fügung. Allerdings gilt diese Risikoabwägung nur, wenn nicht etwa eine äußere Einwirkung wie eine Überspannung, ein Sturz des Speichersystems vom Tisch oder ein Brand- oder Wasserschaden zur Folge hat, dass gleich mehrere in der RAID-Station verwendete Festplattenlaufwerke gleichzeitig zerstört werden.

\section{Die Krux mit dem Datenschutz}

Eine kluge Archivierungsstrategie sieht deshalb auch die räumliche Trennung von Sicherheitskopien und Datenbeständen vor. So kann es sinnvoll sein, eine Kopie der gesicherten Daten auf einem externen Speichermedium nicht in der Praxis, sondern zu Hause oder in einem Bankschließfach zu hinterlegen. Dazu allerdings zwei Hinweise: Zum einen muss natürlich auch eine solche Kopie von Zeit zu Zeit auf ihre Funktionsfähigkeit und Vollständigkeit überprüft werden. Zum anderen sollte der Datenschutz berücksichtigt werden: Patientendaten sind vertrauliche Unterlagen, bei denen Diebstahl oder Verlust sehr unangenehme und aufwändige Konsequenzen haben können. Eine gute Empfehlung zur Absicherung gegen diese Risiken ist eine Verschlüsselung der Daten. Die meisten RAID-Anbieter haben eine Verschlüsselungstechnik gleich mit im Gepäck. Molzen: „Mit Secure Lockware bieten wir ein kostenloses Dienstprogramm zur Verschlüsselung von Daten an. Damit lassen sich komplette Speicherlaufwerke so schützen, dass der Zugriff auf ihre Inhalte nur durch Eingabe eines Kennworts möglich ist.

Hannes Rügheimer

\title{
KBV plant großräumige Vernetzungsoffensive
}

\section{Die Selbstverwaltung sieht die Hoheit über ihr sicheres Online-Netzwerk in Gefahr - und hat ihr Schicksal damit verknüpft.}

$\mathrm{D}$ ie Vernetzung von Ärzten untereinander muss in den Händen der ärztlichen Selbstverwaltung liegen, so will es die KBV. Die Delegierten der KVen gingen sogar so weit, die Hoheit über die "Online-Vernetzung" der Ärzte mit dem Sicherstellungsauftrag zu verknüpfen. Sie solle als achter Punkt die sieben Bedingungen ergänzen, unter denen die Ärzte bereit sind, auch nach 2017 noch den Sicherstellungsauftrag zu erfüllen.

Hintergrund des Vorstoßes aus dem KBV-Vorstand sind Äußerungen eines Managers der Deutschen Telekom. „Im westlichen Europa sind wir das einzige Land, das im Gesundheitswesen auf einen sicheren Online-Austausch von Daten verzichtet. Dabei ist doch längst unstrittig, dass wir eine gute Online-Vernetzung mit einer einheitlichen Infrastruktur und einem hohen Sicherheitsniveau brauchen", hatte der Leiter des Geschäftsfeldes Gesundheit der Telekom, Dr. Axel Wehmeier, in einem Interview für das Branchendossier „Healthcare 2020“ des Marktforschungsinstituts Lünendonk gesagt. Diese Äußerung haben die Ärzte mit Befremden zur Kenntnis genommen. „Gerade die Telekom als zugelassener KV-SafeNet-Provider sollte eigentlich

wissen, dass es bereits eine funktionierende Vernetzung gibt“, wunderte sich KBV-Chef Dr. Andreas Köhler. Das sichere Netz der KVen werde von 35.000 Ärzten und Psychotherapeuten genutzt und werde von den Landesdatenschützern empfohlen.

Köhler unterstellte Wehmeier einen Angriff auf die Souveränität der ärztlichen Selbstverwaltung. Dessen Äußerung, die Telekom wolle „der zentrale Partner für das gesamte Gesundheitswesen sein, der alle Teilnehmer zusammenbringe", mache die Ambitionen des DaxSchwergewichts im Gesundheitswesen deutlich. Köhler brachte stattdessen das sichere Netz der KVen als Alternative zur Netzhoheit der gematik über die Telematik-Infrastruktur im Zusammenhang mit der elektronischen Gesundheitskarte ins Spiel. Der KBV-Vorstand erneuerte die Kritik der Ärzte und Psychotherapeuten an den Plänen der gematik zu dieser Infrastruktur, wandte sich gleichzeitig aber gegen einen Ausstieg aus der Entwicklungsgesellschaft. Die Ärzte sollten die Entwicklung der gematik und der Gesundheitskarte aber weiter begleiten, „um Schlimmeres zu verhindern", so Köhler.

Anno Fricke
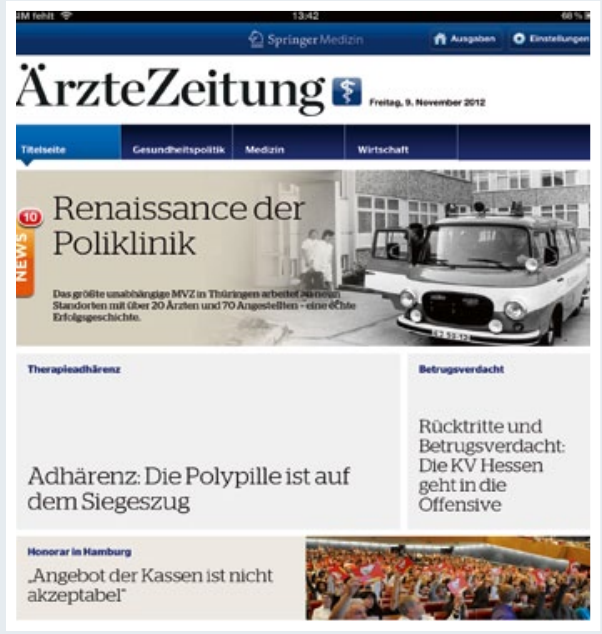

Ärzte Zeitung digital: Heute lesen, was morgen in der Zeitung steht

Im Publikumsbereich erfreuen sich App-Ausgaben von Zeitungen und Zeitschriften wachsender Beliebtheit. Inzwischen ist auch Deutschlands einzige Tageszeitung für Ärzte in diesem Format erhältlich: die „Ärzte Zeitung digital". Die App bietet gesundheitspolitische Nachrichten, Kommentare, Hintergründe zu Medizin und wirtschaftlichen Themen rund um die Praxis - im übersichtlichen und intuitiven Lesemodus, optimiert für das iPad.

Die Vorteile für Leser der App-Ausgabe der "Ärzte Zeitung":

_ Die Ausgabe ist schon am Vorabend verfügbar, Leser können damit die Nachrichten des kommenden Tages bequem auf dem Sofa empfangen.

- Wichtige Nachrichten werden als „Breaking News" direkt auf das Tablet geleitet und lassen sich über die sogenannte Newslasche anzeigen.

- Die App ist so konzipiert, dass die gesamte Ausgabe im Hintergrund heruntergeladen werden kann. Anders als im Internet ist es für Leser dann möglich, sich offline durch die Ausgabe zu „blättern“, ohne Wartezeiten beim Durchklicken auf einer Website.

_ Die alten Ausgaben bleiben zwei Wochen auf dem iPad verfügbar.

„Ärzte Zeitung digital“ ist Teil des e.Med-Pakets von Springer Medizin. Zugänglich ist die App über den iTunes-Store von Apple - allerdings nur für registrierte Nutzer von Springer Medizin, die e.Med für 30 Tage kostenlos testen wollen, oder für e.Med-Abonnenten - und zwar ohne weitere Zusatzkosten. Die Version für Android-Tablets wird Anfang 2013 verfügbar sein.

Anmeldung zum kostenlosen e.Med-Testzugang: www.springermedizin.de/eMed/ Mehr Info zur App „Ärzte Zeitung digital“: www.springermedizin.de/tablet 